\title{
Long-term follow-up after arthroscopic tenotomy for partial rupture of the biceps brachii tendon
}

\author{
A. L. R. Bergenhuyzen; K. A. G. Vermote; H. van Bree; B. Van Ryssen
}

University Ghent Faculty of Veterinary Medicine, Department of Medical Imaging and Small Animal Orthopedics, Merelbeke, Belgium

\author{
Keywords \\ Biceps tendon, arthroscopy, tenotomy, long- \\ term, dog
}

\section{Summary}

Objective: To report the long-term clinical outcomes and radiographic results in dogs diagnosed with partial bicipital rupture and treated by arthroscopic tenotomy.

Materials and Methods: The medical records of dogs that had undergone arthroscopic tenotomy were retrospectively reviewed. Inclusion criteria for this study were: performance of an arthroscopic tenotomy between August 1999 and July 2007, availability of arthroscopic records data for review, and ability to obtain follow-up data for more than one year after arthroscopic tenotomy. In all cases, owners were interviewed during follow-up appointments or via telephone to determine perceived outcome after surgery.

Results: Forty-seven arthroscopic tenotomies were performed on 40 dogs without any

Correspondence to

Dr. Alain Lode Rene Bergenhuyzen

University Ghent Faculty of Veterinary Medicine

Department of Medical Imaging and

Small Animal Orthopedics

Salisburylaan 133

9820 Merelbeke, Oost-Vlaanderen

Belgium

Phone: +32926 47650

Fax: +32926 47739

E-mail: alainbergenhuyzen@hotmail.com major surgical complications. Long-term follow-up examinations, ranging from 12 months to 48 months (mean 26 months) after the tenotomy, were obtained for 24 dogs ( 25 shoulders).

Clinical outcome was assessed as excellent in 22 shoulders, with each dog showing a full return of limb function. A total of 10 dogs (11 joints) were evaluated radiographically; six joints revealed no progression of pathology, and five joints showed a limited progression of pathology.

Conclusion: Arthroscopic tenotomy in the treatment of bicipital partial rupture yields favourable long-term clinical results and a high degree of owner satisfaction. The feasibility of this technique and the long-term clinical and radiographic outcome from our study indicate that this technique can be considered a reliable and safe treatment for partial bicipital rupture.

Vet Comp Orthop Traumatol 2010; 23: 51-55

doi:10.3415/VCOT-09-01-0005

Received: January 14, 2009

Accepted: July 27, 2009

Pre-published online: December 8, 2009 ovitis is complex (6-9). This disease primarily occurs in large or medium-sized dogs of middle-age or older. Clinical signs are usually chronic and progressive (9-11).

Typical clinical findings are a positive biceps test and hyperextension of the elbow along with flexion of the shoulder when a complete bicipital rupture is present (1). Diagnostic imaging for a partially or completely ruptured biceps tendon includes radiography, arthrography, ultrasonography and arthroscopy. Primary radiographic signs are changes at the supraglenoid tubercle, such as osteophytosis, osteosclerosis, radiolucency, or deformation along with calcification and osteophytosis within the tendon sheath. Secondary arthrosis develops with chronicity of the lesion (11). Confirmation of the diagnosis can be made when an ultrasound examination shows a change in the structure of the proximal part of the biceps tendon, as well as defects or a complete rupture. An increased amount of fluid within the tendon sheath is often seen as a secondary sign of synovitis within the joint (12-13). Arthrographic examination can also be used to confirm the diagnosis by demonstrating a changed delineation of the biceps tendon and its sheath (14-16). Arthroscopy allows direct inspection of the biceps tendon, showing thickening of the tendon, ruptured fibres and hyperplastic remnants (17-18). Recently, magnetic resonance imaging has shown great potential as a diagnostic tool in the evaluation of canine shoulder disease, including partial bicipital rupture (19). A correct diagnosis is crucial for obtaining the desired treatment result.

The stabilising function of the biceps tendon in the shoulder remains controversial, therefore treatment varies widely amongst surgeons, ranging from non-operative management to bicipital repair, te-
Tendon and ligament disorders are becoming more frequently recognised as a cause of shoulder lameness in dogs (1-5). Ac- cording to Bardet, the most commonly reported conditions affecting the bicipital tendon are partial or complete tears at its origin (5). The aetiology of partial or complete ruptures, and the associated tenosyn- 
notomy or tenodesis. Surgical treatment is recommended for dogs that do not respond to non-operative management. According to the veterinary literature, good and excellent results have been obtained with both tenotomy and tenodesis, however, the ultimate outcomes were based on preliminary results, and long-term clinical results were unavailable (20-22).

The purpose of this study is to evaluate the long-term clinical outcome and radiographic results in dogs diagnosed with partial bicipital rupture treated by arthroscopic tenotomy.

\section{Materials and methods}

\section{Inclusion criteria}

The medical records of dogs that underwent arthroscopic tenotomy as treatment for partial rupture of the biceps tendon were retrospectively reviewed. Inclusion criteria for this study were: performance of an arthroscopic tenotomy between August 1999 and July 2007, availability of arthroscopic records data for review, and ability to obtain follow-up data for more than one year after the arthroscopic tenotomy.

For this study, diagnosis of partial rupture of the biceps tendon was based on the presence of definitive forelimb lameness lo- calised to the shoulder as well as filling defects of the biceps brachii tendon sheath, abnormal delineation of the tendon as seen on a positive contrast arthrogram, or an amorphous, inhomogeneous, hyperechoic biceps brachii tendon on ultrasonographic examination and confirmed by arthroscopy. Data collected included, age, gender, breed, activity status, orthopaedic examination findings, arthroscopic findings and follow-up examinations.

\section{Arthroscopy}

Shoulder arthroscopy was performed in a standardised manner for all dogs using craniolateral and caudolateral portals (2.7 $\mathrm{mm}, 30^{\circ}$ fore-oblique arthroscope $)^{\mathrm{a}}$ (18). The joint was explored using a standard compartmental approach. The presence of a partial bicipital rupture was assessed by visual examination and probing of the tendon while extending and flexing the elbow. Transection of the tendon was achieved by an arthroscopic hook scissor ${ }^{\mathrm{b}}$. At the time of arthroscopy, digital images of each structure were obtained for subsequent evaluation and data recording.

Richard Wolf GmbH, Knittlingen, Germany

Nr. 98487.04: Richard Wolf Gmbh, Knittlingen

\section{Post-operative care}

The instructions that the owners had received for the first six weeks of postoperative care included short leash walks and restriction of the dog to a small room when unobserved. Analgesics were also administered for three weeks following the surgery, however physiotherapy was not performed. When there was not any evidence of pain at the six week follow-up examination, a progressive return to full activity and non-concussive activities were encouraged over the subsequent six weeks. Unrestricted activity was allowed after 12 weeks of convalescence.

\section{Outcome}

In order to evaluate the treatment outcome, the owners were asked to present their dogs for a clinical and radiological reexamination (Group 1). If the owners could not present their dogs to the clinic again, the treatment was evaluated by means of functional results reported by the owner through a questionnaire ( Supplementary Information available at www. VCOT-online.com) (Group 2). The extent of the remaining complaints and complications were of special interest. All of the dogs presented were observed and video-

\begin{tabular}{|l|l|l|l|l|l}
\hline \multicolumn{7}{c}{ Clinical outcome and radiographic data for Group 1 } \\
\hline & $\begin{array}{l}\text { Age at first } \\
\text { presentation } \\
\text { (months) }\end{array}$ & $\begin{array}{l}\text { Preoperative } \\
\text { radiographic } \\
\text { osteoarthritis }\end{array}$ & $\begin{array}{l}\text { Postoperative } \\
\text { radiographic } \\
\text { osteoarthritis }\end{array}$ & $\begin{array}{l}\text { Clinical } \\
\text { outcome }\end{array}$ & $\begin{array}{l}\text { Time to } \\
\text { follow-up } \\
\text { (months) }\end{array}$ \\
\hline Breed & 73 & Grade I & Grade I & Excellent & 14 \\
\hline Bewfoundland & 83 & Grade I & Grade II & Poor & 32 \\
\hline Beauceron & 52 & Grade II & Grade II & Excellent & 22 \\
\hline Border Collie & 53 & Grade I & Grade II & Excellent & 22 \\
\hline Border Collie & 54 & Grade I & Grade I & Excellent & 20 \\
\hline Border Collie & 45 & Grade II & Grade III & Good & 31 \\
\hline Bernese Mountain dog & 10 & Grade I & Grade II & Excellent & 43 \\
\hline Bernese Mountain dog & 23 & Grade I & Grade I & Excellent & 14 \\
\hline Bernese Mountain dog & 45 & Grade I & Grade I & Excellent & 24 \\
\hline Rottweiler & 43 & Grade I & Grade II & Excellent & 31 \\
\hline Irish Wolf Hound & 78 & Grade I & Grade I & Excellent & 13 \\
\hline
\end{tabular}

Table 1

Clinical, radiographical and follow-up findings of the dogs that were represented for clinical and radiographic reevaluation (Group 1). 
recorded walking and trotting on leash, in a straight line, and in a circle (two directions). Two investigators (AB, BR) independently assigned an individual lameness score for each forelimb of each dog. The lameness score was based on a 5-point scale: $0=$ no detectable lameness, $1=$ mild weight-bearing lameness, $2=$ moderate weight-bearing lameness, $3=$ marked weight-bearing lameness, 4 = non-weightbearing lameness. The evaluation of the treatment's success was based on the findings of the clinical examination and on the owners' reports (questionnaires). The outcome was rated as 'excellent' when no lameness was observed or reported in all activities and the use of anti-inflammatory medication was not required. The outcome was rated 'good' in dogs with occasional lameness or exercise intolerance that was sensitive to anti-inflammatory medication. All other outcomes were rated 'poor'.

Radiographs from the initial examination and the follow-up examination were evaluated and compared with respect to subchondral bone sclerosis, remodelling, osteophytosis and enthesiophytosis. The joint was then subjectively graded based on the severity of the radiographic severity of osteoarthritis ( Fig. 1).

\section{Results}

\section{Patients}

Forty-seven arthroscopic tenotomies were performed in 40 dogs, but only 24 dogs met the inclusion criteria due to lack of followup. Nine dogs were no longer living at the time of owner contact, thus an interview was not performed. Contact data were not available for the owners of seven other dogs because of address change. Of the 24 dogs that met the inclusion criteria, all were large breed and included: Bernese Mountain Dog $(\mathrm{n}=7)$, Newfoundland $(\mathrm{n}=2)$, Golden Retriever $(\mathrm{n}=3)$, Border Collie ( $\mathrm{n}$ $=3)$, Crossbreed $(n=2)$, Rottweiler $(n=1)$, American Staffordshire Terrier $(\mathrm{n}=1)$, Labrador Retriever $(\mathrm{n}=1)$, German Shepard $(\mathrm{n}=1)$, Irish Wolf Hound $(\mathrm{n}=1)$, Beauceron $(n=1)$, and Nova Scotia Duck-Tolling Retriever $(n=1)$. The dogs' ages ranged from six months to 10 years (mean: 3.5
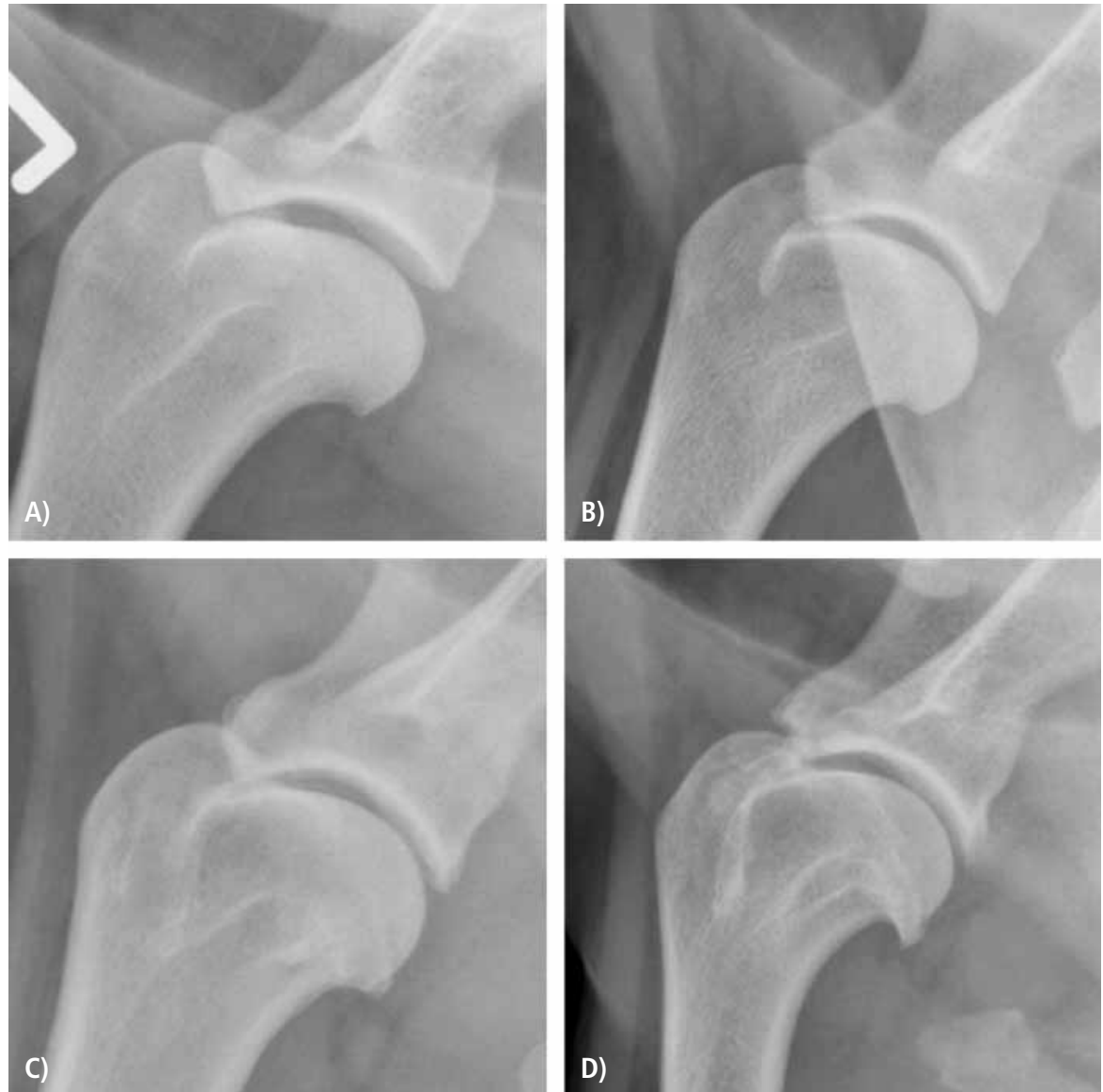

Fig. 1 Joints with partial rupture of the biceps tendon shown at different degrees of osteoarthritis. A) Grade 0 - No radiographic abnormality; B) Grade 1 - Sclerosis at the glenoid rim and medial trochlea of the biciptal sulcus; C) Grade 2 - Osteophytosis less than $3 \mathrm{~mm}$ at the caudal rim of the humeral head; D) Grade 3 - Osteophytosis more than $3 \mathrm{~mm}$ at the caudal rim of the humeral head.

years). The group included 10 entire females, one spayed female, nine entire males, and four neutered males.

\section{Clinical and arthroscopic findings}

The degree of lameness varied among the dogs $(n=24)$, ranging from subtle, chronic intermittent lameness to permanent nonweight-bearing lameness. The duration of lameness ranged from three weeks to three years (mean: 3.5 months). Only in half of the cases, was there a history of trauma and acute onset of lameness. Orthopaedic examination findings included atrophied shoulder muscles $(n=22)$, a painful shoulder in extension $(\mathrm{n}=5)$, a positive biceps test $(\mathrm{n}=21)$ and hyperextension of the elbow $(n=2)$. Besides the ruptured aspect of the biceps tendon, the most consist- ent arthroscopic findings were synovial hypertrophy and hyperaemia $(n=21)$, and fibrillation of the medial glenohumeral ligament $(n=8)$. Other findings included fibrillation of articular cartilage of the humeral head $(\mathrm{n}=3)$, fibrillation of the subscapularis muscle $(n=2)$, and a partial rupture of the medial glenohumeral ligament $(n=1)$.

\section{Outcome and radiographic evaluation}

Long-term follow-up examinations were obtained for 24 dogs ( 25 shoulders) and ranged from 12 months to 48 months (mean: 26 months). No complications related to the tenotomy were reported. The follow-up exam consisted of a second clinical and radiological examination of the af- 
fected dogs (Group 1; $\mathrm{n}=10$ dogs [11 shoulders]) or a questionnaire answered by the dog's owner (Group 2; $\mathrm{n}=14$ dogs [14 shoulders]). In Group 1, nine out of 10 dogs ( 11 shoulders) showed an excellent result, with relief of symptoms at a mean time of three weeks. The dogs did not have any abnormalities on clinical examination, were without lameness at the time of the long-term follow-up examination, and did not show any signs of lameness after exercise. The result for one dog was rated as 'good' (lameness score 1) as the dog showed no signs of lameness for the majority of the time; however, after hard exercise or when running in circles, a low degree of lameness was noticed. One result was rated as 'poor' as the dog suffered from permanent low to moderate lameness. A second arthroscopic examination revealed fibrous tissue in the inter-tubercular groove which originated from the supraglenoidal tubercle. The lameness did not improve after the second arthroscopic transection of the fibrous tissue. Despite the latter case, none of the dogs developed an abnormal gait or an inability to flex the elbow. In Group 2, 13 out of 14 of the results obtained via the questionnaire were rated as 'excellent', and one report was rated as 'good'. No cosmetic deformities were noticed in Group 1 or in Group 2.

A total of 10 dogs (11 joints) were evaluated radiographically and these examinations did not reveal any progression of pathology in six joints. Five joints showed a limited progression of pathology. The median progression was 0 ( $\$$ Table1).

\section{Discussion}

The goal of surgical treatment in cases of partial bicipital rupture is to eliminate movement of the tendon in the inflamed tendon sheath. This can be accomplished with either tenodesis or tenotomy. Initially, the biceps tenotomy procedure was criticised due to bio-mechanical data and a lack of long-term clinical results (22-24). The canine biceps brachii muscle is one of many musculo-tendinous units that cross two joints. In the elbow, it serves as a flexor and supinator. Whilst its function at the elbow is clear, its role in the shoulder remains controversial. An in vitro study has confirmed that the biceps tendon contributes to the passive shoulder stability, particularly in the neutral and flexed positions (24). However, scapulo-humeral stability after bicipital tenotomy should be comparable to that after a traditional bicipital tenodesis. If significant instability had been present after tenotomy, we would have expected to see signs of clinical, and radiographic abnormalities. In the authors' opinion, the biceps tendon does not have a primary stabilising function in the shoulder, but has multiple secondary roles instead. Therefore, as no primary function can be isolated, it is not surprising that there is no single reliable clinical test or treatment for biceps pathology.

In human medicine, tenodesis was initially advised to re-establish the resting muscle length and thereby maintain the length-tension relationship, prevent muscle atrophy, avoid cramping pain, maintain elbow flexion and supination strength, and avoid cosmetic deformity (Popeye sign) (25). Currently, tenotomy is becoming more popular than tenodesis (26-28). Mariani and colleagues compared 30 patients with spontaneous rupture of the long head of the biceps treated non-operatively with 26 patients who underwent early biceps tenodesis (29). They found only a $13 \%$ difference in the biceps' supination strength between the two groups and no difference at all in elbow flexion strength. However, in patients who are concerned about potential cosmetic deformity and associated dysfunction, tenodesis might be advantageous. It is important to note that none of the dogs in this study developed an abnormal gait, a cosmetic deformity or the inability to flex the elbow.

Pre-operative clinical findings in this study are similar to those reported in the literature, however in two patients, hyperextension of the elbow was possible, despite the fact that the biceps tendon was not completely ruptured.

Clinical outcome was excellent in 22 shoulders, with each dog showing a full return of limb function. Symptoms disappeared rapidly in the majority of the dogs, with three weeks being the mean. Since most dogs showed signs of immediate pain relief, it is likely that tenotomy eliminated the painful traction forces that were exerted on the non-ruptured part of the affected biceps tendon, and its attachment onto the supraglenoid tubercle. In the case that had a recurrence of lameness and a second arthroscopic examination, we believe that this was the result of an incomplete tenotomy of the biceps tendon in the initial arthroscopy. During arthroscopic tenotomy, the tendon does not always retract clearly in its groove, which can make it difficult to judge whether or not the tendon is completely transected, particularly in chronic cases.

Fibrillation of the medial glenohumeral ligament present in eight out of 25 joints, and a partial rupture of the medial glenohumeral ligament present in one out of 25 joints were regarded as secondary findings. Postoperative exercise restriction may have contributed to the recovery because the outcome was rated 'excellent' in all affected dogs. Therefore, we recommend caution in interpreting such findings as a primary clinical problem, because, in our opinion, some could be coincidental findings of no clinical significance. Although limited, osteoarthritis was present preoperatively in all dogs from Group 1. We could not demonstrate a correlation between presence of increase in grade and the clinical outcome. However, all dogs with postoperative grade I (5 out of 11) had excellent clinical outcomes. Dogs showing clinical signs for more than five weeks were associated with higher postoperative grades, which suggests that early recognition and treatment could produce better clinical outcomes. The number of radiographic cases was not large enough for statistical analysis.

A major limitation of our study is that tenodesis (open or arthroscopically) was not evaluated as an alternative treatment. As no comparison was made, tenotomy cannot be considered superior to tenodesis based on the available data. Long-term studies designed to evaluate tenodesis have not yet been made.

Another limitation is that dogs from Group 2 were evaluated only by a subjective questionnaire. Force plate analysis, computed tomography and arthroscopy may have contributed important data, but they were beyond the scope of this study. Indeed, owners are not inclined to come for an additional examination if no problems 
are present and this could have biased our results - even positively - because, for the majority of the dogs, the short-term results (which are not included in this study) were rewarding.

We conclude that arthroscopic tenotomy in the treatment of bicipital partial rupture yields favourable long-term clinical results and a high degree of owner satisfaction. Although it cannot be considered superior to tenodesis, the feasibility of applying this technique, and the long-term clinical and radiographic outcomes from our study suggest that this technique can be considered a safe, reliable treatment for partial bicipital tendon rupture.

\section{References}

1. Bardet JF. Shoulder diseases in dogs. Vet Med 2002; 12: 909-918

2. Kramer M, Schimke E, Gerwing M, et al. Bizepssehnenerkrankungen beim Hund als Lahmheitsursache. Der praktische Tierarzt 1997; 78: 274-292.

3. Innes JF, Brown G. Rupture of the biceps brachii tendon sheath in two dogs. J Small Anim Pract 2004; 45: 25-28.

4. Bardet JF. Diagnosis of shoulder instability in dogs and cats: a retrospective study. J Am Anim Hosp Assoc 1998; 34: 42-54.

5. Bardet JF. Lesions of the biceps tendon: Diagnosis and classification. Vet Comp Orthop Traumatol 1999; 12: 188-195.

6. Lincoln ID, Potter K. Tenosynovitis of the biceps brachii tendon in dogs. J Am Anim Hosp Assoc 1984; 20: 385-392.

7. Davidson EB, Griffey SM, Vasseur PB, et al. Histopathological, radiographic, and arthrographic comparison of the biceps tendon in normal dogs and dogs with biceps tenosynovitis. J Am Anim Hosp Assoc 2000; 36: 522-530.

8. Gilley RS, Wallace LJ, Hayden DW. Clinical and pathologic analyses of bicipital tenosynovitis in dogs. Am J Vet Res 2002; 63: 402-407.

9. Stobie D, Wallace LJ, Lipowitz AJ, et al. Chronic bicipital tenosynovitis in dogs: 29 cases (1985-1992). J Am Vet Med Assoc 1995; 207: 201-207.

10. Piermattei DL, Flo GL. The shoulder joint: Handbook of Small Animal Orthopedics and Fracture Treatment (3rd ed). Brinker WO, Piermattei DL, Flo GL (Editors). Philadephia: Saunders 1997: 228-260.

11. Lechleitner E, Mayrhofer E. Tendopathien der Ursprungssehne des M.biceps brachii beim Hund röntgenologische, pathoanatomische and pathohistologische Befunde. Kleintierpraxis 1993; 38: 161-178.

12. Kramer M, Gerwing M, Hach V, et al. Sonography of the musculoskeletal system in dogs and cats. Vet Radiol Ultrasound 1997; 38: 139-149.

13. Kramer M, Gerwing M, Sheppard C, et al. Ultrasonography for the diagnosis of diseases of the tendon and tendon sheath of the biceps brachii muscle. Vet Surg 2001; 30: 64-71.

14. Barthez PY, Morgan JP. Bicipital tenosynovitis in the dog - evaluation with positive contrast arthrography. Vet Radiol Ultrasound 1993; 34: 325-330.

15. van Bree H. Evaluation of the prognostic value of positive-contrast shoulder arthrography for bilateral osteochondrosis lesions in dogs. Am J Vet Res 1990; 51: 1121-1125.

16. van Bree H. Comparison of the diagnostic accuracy of positive-contrast arthrography and arthrotomy in evaluation of osteochondrosis lesions in the scapulohumeral joint in dogs. J Am Vet Med Assoc 1993; 203: 84-88.

17. van Bree H, van Ryssen B. Arthroscopy in the diagnosis and treatment of front leg lameness. Am J Vet Res 1995; 17: Suppl. 1, S32-S34.

18. van Ryssen B, van Bree H, Vyt P. Arthroscopy of the shoulder joint in the dog. J Am Anim Hosp Assoc 1993; 29: 101-105.
19. Murphy SE, Ballegeer EA, Forrest LJ, et al. Magnetic resonance imaging findings in dogs with confirmed shoulder pathology. Vet Surg 2008; 37: 631-638.

20. Wall CR, Taylor R. Arthroscopic biceps brachii tenotomy as a treatment for canine bicipital tenosynovitis. J Am Anim Hosp Assoc 2002; 38: 169-175.

21. Adamiak Z, Szalecki P. Treatment of bicipital tenosynovitis with double tenodesis. J Small Anim Pract 2003; 44: 539-540.

22. Cook JL et al. Arthoscopic biceps tenodesis: Technique and six results in six dogs. J Am Anim Hosp Assoc 2005; 41: 121-127.

23. Wolfe RS, Zheng N, Weichel D. Long head of biceps tenotomy versus tenodesis: a cadaveric biomechanical analysis. Arthroscopy 2005; 21: 182-185.

24. Sidaway BK, McLaughlin RM, Elder SH, et al. Role of the tendons of the biceps brachii and infraspinatus muscles and the medial glenohumeral ligament in the maintenance of passive shoulder joint stability in dogs. Am J Vet Res. 2004 (Sep); 65:1216-1222.

25. Osbahr DC, Diamond AB, Speer KP. The cosmetic deformity of the biceps muscle after long head tenotomy versus tenodesis. Arthroscopy: JArthorosco Relat Surg 2002; 18: 483-487.

26. Gill TJ, McIrvin E, Mair SD, et al. Results of biceps tenotomy for treatment of pathology of the long head of the biceps brachii. J Shoulder Elbow Surg 2001; 10: 247-249.

27. Walch G, Edwards TB, Boulahia A, et al. Arthroscopic tenotomy of the long head of biceps in the treatment of rotator cuff tears: clinical and radiographic results of 307 cases. J Shoulder Elbow Surg 2005; 14: 238-246.

28. Maynou C, Mehdi N, Cassagnaud X, et al. Clinical results of arthroscopic tenotomy of the long head of the biceps brachii in full thickness tears of the rotator cuff without repair: 40 cases. Rev Chir Orthop Reparatrice Appar Mot 2005; 91: 300-306.

29. Mariani EM, Cofield RH, Askew LJ, et al. Rupture of the tendon of the long head of the biceps brachii surgical versus non surgical treatment. Clin Orthop Relat Res 1988; 228: 233-239. 